\title{
Differential rotation and angular momentum
}

\author{
G. J. J. Botha ${ }^{1}$ and E. A. Evangelidis ${ }^{2}$ \\ ${ }^{1}$ Department of Applied Mathematics, University of Leeds, Leeds LS2 9JT, UK \\ email: gert@maths.leeds.ac.uk \\ ${ }^{2}$ Department of Environmental Engineering, Laboratory of Non-Conventional Sources of \\ Energy, Demokritos University of Thrace, Kimeria, Xanthi 67100, Greece \\ email: eevangel@env.duth.gr
}

\begin{abstract}
Differential rotation not only occurs in astrophysical plasmas like accretion disks, it is also measured in laboratory plasmas as manifested in the toroidal rotation of tokamak plasmas. A re-examination of the Lagrangian of the system shows that the inclusion of the angular momentum's radial variation in the derivation of the equations of motion produces a force term that couples the angular velocity gradient with the angular momentum. This force term is a property of the angular velocity field, so that the results are valid wherever differential rotation is present.
\end{abstract}

Keywords. Accretion disks, plasmas

\section{Introduction}



Figure 1. A reference frame with origin at the centre of curvature $(\mathrm{CC})$, rotating in an anti-clockwise direction.
In the analysis of motion of a charged particle on a magnetic field line, Alfvén showed the existence of a force

$$
\mathbf{f}=-\frac{m v_{\perp}^{2} \kappa}{2} \mathbf{N}
$$

where $\mathbf{N}$ is the first normal of the co-moving trihedral given by $(\mathbf{T}, \mathbf{N}, \mathbf{b})$ and $\kappa$ is the curvature. We use the original notation of the Frenét system of differential geometry, where $\mathbf{T}$ is the unit vector along the magnetic field and $\mathbf{b}$ the binormal of the orthogonal system (Figure 1). In a reference frame located at the centre of curvature (CC) and rotating with an angular velocity $\boldsymbol{\Omega}$, a particle at point $P$ and moving in a circular orbit, develops a centrifugal force $-m \Omega^{2} \rho \mathbf{N}=-\left(m v_{\|}^{2} / \rho\right) \mathbf{N}$ where $\rho$ is the distance from $P$ to the origin of the reference frame. This expression, when combined with the force described in equation (1.1), gives the total force

$$
\mathbf{f}_{t}=-\frac{m v_{\perp}^{2} \kappa}{2} \mathbf{N}-\frac{m v_{\|}^{2}}{\rho} \mathbf{N}=-m \kappa\left(\frac{v_{\perp}^{2}}{2}+v_{\|}^{2}\right) \mathbf{N} .
$$

In a rotating reference system there exists also the Coriolis force (Sivukhin 1965). The force acting on a charge at point $P$ in a general magnetic field produces the drift velocity

$$
\mathbf{v}_{d}=\frac{\mathbf{f}_{t} \times \mathbf{B}}{e B^{2}}=\frac{1}{e B^{2}} \mathbf{f}_{t} \times\left(B_{T}, B_{N}, B_{b}\right)=\frac{m \kappa}{e B^{2}}\left(\frac{v_{\perp}^{2}}{2}+v_{\|}^{2}\right)\left(-B_{b}, 0, B_{T}\right) .
$$

It is well known that accretion disks follow a Keplerian rotational profile. The analysis presented here will generalize the above results by considering the dynamical problem of motion of a particle in a rotating reference frame with variable angular velocity. 


\section{Rotating reference frame}

In a rotating reference frame the Lagrangian of a moving particle takes on the form (Landau \& Lifshitz 1976)

$$
L=\frac{1}{2} m v^{2}+m \mathbf{v} \cdot \boldsymbol{\Omega} \times \mathbf{r}+\frac{1}{2} m(\boldsymbol{\Omega} \times \mathbf{r})^{2}-m \mathbf{W} \cdot \mathbf{r}-U,
$$

where the velocity in an inertial reference frame

$$
\mathbf{v}_{0}=\mathbf{v}+\mathbf{V}+\mathbf{\Omega} \times \mathbf{r}
$$

is composed of the particle velocity $\mathbf{v}$ in the moving reference frame and the translational and rotational part of the moving frame's velocity, with $\boldsymbol{\Omega} \times \mathbf{r}$ the velocity associated with the rotation and $\mathbf{V}$ the translational velocity. The particle has mass $m$ and position vector $\mathbf{r}$. $U$ is the potential energy and $\mathbf{W}=d \mathbf{V} / d t$ is the translational acceleration of the moving reference frame. In the rest of this discussion we shall ignore the term containing the translational acceleration. To obtain the equation of motion

$$
\frac{d}{d t}\left(\frac{\partial L}{\partial \mathbf{v}}\right)=\frac{\partial L}{\partial \mathbf{r}}
$$

we need to calculate the derivatives with respect to the velocity and position vectors. Here we differ from the usual analysis in that the angular velocity varies with the radial distance, that is, the system is sheared. Thus,

$$
\begin{aligned}
& \frac{\partial L}{\partial \mathbf{v}}=m(\mathbf{v}+\boldsymbol{\Omega} \times \mathbf{r}), \\
& \frac{\partial L}{\partial \mathbf{r}}=m[(\nabla \boldsymbol{\Omega}) \times \mathbf{r}] \cdot(\mathbf{v}+\boldsymbol{\Omega} \times \mathbf{r})+m(\mathbf{v} \times \boldsymbol{\Omega})+m \boldsymbol{\Omega} \times(\mathbf{r} \times \boldsymbol{\Omega})-\nabla U
\end{aligned}
$$

so that the equation of motion (2.3) becomes

$$
m \frac{d \mathbf{v}}{d t}=\mathbf{f}_{\Omega}+\mathbf{f}_{\nabla \Omega}+m \mathbf{r} \times \dot{\Omega}-\nabla U
$$

with

$$
\begin{aligned}
\mathbf{f}_{\Omega} & =m[2 \mathbf{v} \times \boldsymbol{\Omega}+\boldsymbol{\Omega} \times(\mathbf{r} \times \boldsymbol{\Omega})], \\
\mathbf{f}_{\nabla \Omega} & =m[[(\nabla \boldsymbol{\Omega}) \times \mathbf{r}] \cdot(\mathbf{v}+\boldsymbol{\Omega} \times \mathbf{r})] .
\end{aligned}
$$

The two terms in $\mathbf{f}_{\Omega}$ are the Coriolis and centrifugal forces, while the last term in (2.6) is the force field. The term containing $\dot{\Omega}$ is zero if the rotation does not change with time. (We shall assume this to be the case in the subsequent analysis.) In order to analyze the terms in $\mathbf{f}_{\nabla \Omega}$ whose existence depend on the shear (i.e. $\nabla \boldsymbol{\Omega}$ ), we write them in component form. In cylindrical coordinates $(\rho, \phi, z)$ with $\boldsymbol{\Omega}=\Omega \hat{\mathbf{e}}_{z}$, we obtain

$$
\begin{aligned}
{[(\nabla \boldsymbol{\Omega}) \times \mathbf{r}] \cdot \mathbf{v} } & =\rho^{2} \dot{\phi} \nabla \Omega, \\
{[(\nabla \boldsymbol{\Omega}) \times \mathbf{r}] \cdot(\boldsymbol{\Omega} \times \mathbf{r}) } & =\rho^{2} \Omega \nabla \Omega,
\end{aligned}
$$

where $\Omega=|\Omega|$. Using velocity (2.2), it follows from the definition of angular momentum $\mathbf{M}=m \mathbf{r} \times \dot{\mathbf{r}}$ that its $z$ component in the inertial reference frame can be written as

$$
M_{z}=m \rho^{2}(\dot{\phi}+\Omega) \text {. }
$$

Therefore,

$$
\mathbf{f}_{\nabla \Omega}=M_{z} \nabla \Omega
$$


so that equation (2.6) becomes

$$
\frac{d \mathbf{p}}{d t}=\mathbf{f}_{\Omega}+M_{z} \nabla \Omega-\nabla U
$$

where $p$ is the linear momentum of the moving particle. We observe that a radial force term appears due to the coupling of the angular momentum and the gradient of the differential rotation. In tokamak plasmas $\nabla \Omega<0$ in the outer parts of the plasma column, so that the radial force is directed inward in these parts. For $\Omega$ radially increasing, as for accretion disks, the force is directed outwards. We conclude that this force is a property of the $\Omega$ field - independent of the particle motion - as can be seen by eliminating the angular motion of the plasma particles $(\dot{\phi}=0)$.

\section{Guiding centre motion}

Equation (2.13) can be written as

$$
m \frac{d \mathbf{v}}{d t}=\mathbf{f}_{\Omega}+\mathbf{f}_{\nabla \Omega}-\nabla U
$$

where $\mathbf{f}_{\Omega}$ is described by (2.7) and $\mathbf{f}_{\nabla \Omega}$ by (2.12). The terms in $\mathbf{f}_{\Omega}$ are well known in the literature, with the first term the Coriolis force and the second term the centrifugal force. The latter can be written as

$$
\boldsymbol{\Omega} \times(\mathbf{r} \times \boldsymbol{\Omega})=\Omega^{2} \mathbf{r}-(\boldsymbol{\Omega} \cdot \mathbf{r}) \boldsymbol{\Omega}=\rho \Omega^{2} \hat{\mathbf{e}}_{\rho}
$$

which is the centrifugal term $\left(v_{\|}^{2} / \rho\right)(-\mathbf{N})$ included in $(1.2)$. The terms in $\mathbf{f}_{\Omega}$ are

$$
\mathbf{f}_{\Omega}=-2 m \Omega \dot{\rho} \hat{\mathbf{e}}_{\phi}+m \rho \Omega(2 \dot{\phi}+\Omega) \hat{\mathbf{e}}_{\rho},
$$

so that the equation of motion (3.1) can be written as

$$
\frac{d \mathbf{p}}{d t}=\mathbf{f}_{t o t}=-2 m \Omega \dot{\rho} \hat{\mathbf{e}}_{\phi}+m \rho \Omega(2 \dot{\phi}+\Omega) \hat{\mathbf{e}}_{\rho}+m \rho^{2}(\dot{\phi}+\Omega) \nabla \Omega-\nabla U
$$

If we assume rotational symmetry, i.e. $\nabla \Omega=(\partial \Omega / \partial \rho) \hat{\mathbf{e}}_{\rho}$, then the total force in the equation of motion reduces to

$$
\mathbf{f}_{t o t}=-2 m \Omega \dot{\rho} \hat{\mathbf{e}}_{\phi}+m \rho\left[(2 \dot{\phi}+\Omega) \Omega+\rho(\dot{\phi}+\Omega) \frac{\partial \Omega}{\partial \rho}\right] \hat{\mathbf{e}}_{\rho}-\nabla U .
$$

Neglecting $\nabla U$, the guiding centre drift in a magnetic field due to this force is

$$
\mathbf{v}_{d}=\frac{2 m \dot{\rho} \Omega}{e B^{2}}\left(-B_{z}, 0, B_{\rho}\right)+\frac{m \rho}{e B^{2}}\left[(2 \dot{\phi}+\Omega) \Omega+\rho(\dot{\phi}+\Omega) \frac{\partial \Omega}{\partial \rho}\right]\left(0,-B_{z}, B_{\phi}\right),
$$

with $e$ the charge of the particle under consideration. In tokamaks $B_{\phi}$ is dominant, so that (3.6) reduces to motion along the rotational axis. In the case of accretion disks, a dominant axial magnetic field $\left(B_{z}\right)$ reduces $(3.6)$ to motion in the rotational plane. Apart from drift due to particle motion (i.e. the $\dot{\rho}$ and $\dot{\phi}$ terms) there exists azimuthal drift due to the differential rotation of the reference system.

\section{References}

Landau, L.D. \& Lifshitz, E.M. 1976, Mechanics, Course of Theoretical Physics, (Oxford: Pergamon Press) vol. 1, par. 39

Sivukhin, D.V. 1965, in: M.A. Leontovich (ed.), Reviews of Plasma Physics, (New York: Consultants Bureau), vol. 1, p. 31 